\title{
Gaucher Disease Screening at a General Adult Haematology Referral, Single Tertiary Care Centre: A Prospective Study
}

Steven A Russell ${ }^{1 *}$, Mike Keeney², Ben Hedley², Margo Bode ${ }^{2}$, Joy Mangel ${ }^{3}$, Alan Gob $^{3}$, Selay Lam ${ }^{3}$, Chai Phua ${ }^{3}$, Cyrus C Hsia ${ }^{3}$ and Michelle Sholzberg ${ }^{4}$

${ }^{1}$ Department of Internal Medicine, Western University, Canada

${ }^{2}$ London Laboratory Services Group, London, UK

${ }^{3}$ Division of Hematology, Department of Medicine, Western University, Canada

${ }^{4}$ Department of Medicine and Laboratory Medicine \& Pathobiology, St. Michael's Hospital, Li Ka Shing Knowledge Institute, University of Toronto, Canada

*Corresponding author: Steven A Russell, Division of Internal Medicine, Western University, Canada, N6A 5W9, Tel: 1-519-685-8500 x 56060; Fax: 1-519-685-8294; Email: steverussell24@gmail.com

Received date: June 05, 2018; Accepted date: June 18, 2018; Publication date: June 25, 2018

Copyright: (c) 2018, Russell SA, et al. This is an open-access article distributed under the terms of the Creative Commons Attribution License, which permits unrestricted use, distribution, and reproduction in any medium, provided the original author and source are credited.

\begin{abstract}
Background: Gaucher Disease (GD) is the most common hereditary lysosomal storage disease where glucocerebrosidase deficiency leads to glycolipid accumulation in macrophages. Given its non-specific symptoms, GD may be under-recognized by physicians.
\end{abstract}

Objective: The goal of our study is to prove the feasibility of screening high risk patient for GD.

Participants and Design: In 1 year, 221 patients with thrombocytopenia and/or splenomegaly from three benign hematology clinics (three half-days per week) were prospectively enrolled and screened for GD using a validated high-throughput fluorescent dried blood spot assay.

Key Results: Of the 221patients tested, 11 had a positive initial screening test and two were confirmed heterozygotes for the gene encoding $\beta$-glucocerebrosidase.

Conclusion: This study has demonstrated the feasibility of screening high risk patients for GD. Expanding to other centres, this number could increase substantially allowing for a larger sample size to power exploration of additional hypotheses.

Keywords: Gaucher Disease; Dried blood spot assay

\section{Introduction}

Gaucher Disease (GD) is the most common hereditary lysosomal storage disease where glucocerebrosidase deficiency leads to glycolipid accumulation in macrophages [1]. The result is spleen, liver, bone, and central nervous system (CNS) overcrowding by glycolipid-laden macrophages $[2,3]$. Of the three types of Gaucher Disease, Type 1 (GD1) accounts for $94 \%$ of cases and can present in adulthood. The prevalence of GD1 is 1 in 75,000 births [1]. However, in people of Ashkenazi Jewish descent prevalence rates are substantially increased at 1 in 600 [1]. GD has a genotype-phenotype correlation where both homozygotes and heterozygotes can have clinical disease [1]. From the International Gaucher Registry in 2008, which comprises nearly 5000 GD patients, $86 \%$ had splenomegaly and $60 \%$ had thrombocytopenia [4]. Despite these relatively common clinical and laboratory features, the diagnosis is frequently overlooked secondary to historically difficult diagnostic tests and no previous effective treatment. A recently published study by Motta et al. [5] designed an observational study to screen high pre-test probability patients in Italy. Screening patients with splenomegaly and/or thrombocytopenia presenting to haematology units revealed a nearly $4 \%$ prevalence of GD1 in this Italian population [5]. The overarching aim of our study was to replicate the Italian screening method on a Canadian multi-ethnic population to estimate the point prevalence of GD1 in Canadian patients referred to general haematology clinics. Thus, the primary objective was to assess the feasibility of using the same screening test by Motta et al. [5] in a general adult Haematology clinic with the ultimate goal of expanding the study to more Canadian centres to get an estimate of the point prevalence of GD in the Canadian population.

\section{Methods}

\section{Study design}

Study enrolment started in November, 2015 and closed in November, 2016. Patients were enrolled from one of three benign haematology clinics within a single-centre tertiary care Canadian hospital. Inclusion criteria were patients with splenomegaly (confirmed with ultrasound, computed tomography, or magnetic resonance imaging) and/or thrombocytopenia (platelet count $<150 \times 10^{3} / \mathrm{mm}^{3}$ ). History and physical examination focused on bleeding and fracture history, symptoms of splenic enlargement, and bone pain. Demographic characteristics such as age, gender, ethnicity, and body mass index (BMI) were collected. Only blood-work and imaging obtained by the most responsible treating physician (MRP) was compiled. 


\section{Dried blood spot and laboratory tests}

The Dried Blood Spot (DBS)-based approach to screen for GD has been previously validated by Genzyme Corporation [6]. The DBS test is a high-throughput fluorescent assay developed using the $\beta$-Dglucosidase (GBA) substrate 4-methylumbelliferone (4-MU) and a fixed filter paper based matrix. Venous blood was drawn from each patient and $75 \mu \mathrm{l}$ aliquots were spotted onto filter-paper based matrices. Bloodspots were then dried. One $3.2 \mathrm{~mm}$ disc was punched from each bloodspot and allowed to react with artificial 4-MU in a buffered solution ( $\mathrm{pH}$ 5.2) for 20 hours at 37 degree Celsius. Fluorescence of 4-MU is $355 \mathrm{~nm}$. After being enzymatically processed by GBA, its peaked emission wavelength becomes $460 \mathrm{~nm}$. Thus, measuring fluorescence emission wavelengths of each dried-blood spot disc allows indirect assessment of the level of functional $\beta$-Dglucosidase in each patient's venous blood sample. Validation involved testing 60 normal patients. The mean activity was $6.67 \mathrm{pmol} / \mathrm{punch} /$ hour. From this sample, the lowest 2.5 percentile was set as the cut-off for a positive test $(<3.42 \mathrm{pmol} / \mathrm{punch} / \mathrm{hour})$. Borderline results were classified as $30 \%$ above the cut-off value (3.43-4.45 pmol/punch/hour). Tests were run in duplication at two substrate concentrations. Additionally, a negative control is performed with the same sample run in the presence of an inhibitor for glucocerebrosidase (conduritol B epoxyde). Results are validated for sample degradation by testing for another enzymatic reaction (e.g. alpha-galactosidase A for Fabry disease). If the second enzyme activity is reduced then the sample is consider degraded and another sample is requested. Borderline values were repeated. A result of $<4.45 \mathrm{pmol} /$ punch/hour (classified as borderline or positive test) has a sensitivity and specificity of $88.2 \%$ and $88.5 \%$ respectively [7]. When patients were enrolled in our study the central laboratory for the haematology clinic would spot enrolled patients' blood onto collection cards provided within 24 hours. Sample would dry for 1 hour and was kept at room temperature before being shipped to one Dynacare laboratory that was capable of adequate processing and interpreting the results. With a normal range of DBS GBA activity ranging from 4.4-17.7 pmol/punch/hr, participants with positive or borderline results had this information communicated to their MRP for disclosure. The protocol for an initial positive screen included a repeat DBS, leukocyte enzyme activity, and PCR testing of the gene encoding $\beta$-glucocerebrosidase. Confirmed GD patients would go on to receive the standard of care, including bone marrow biopsy, bone MRI, genetic counselling and referral to a physician with expertise managing GD.

\section{Statistical Analysis}

\section{Sample size calculation}

We estimated that we would require a total of 369 patients to be enrolled in this study. The sample size calculations for $95 \%$ confidence level assuming a true population prevalence rate of $4 \%+/-2 \%$ like in the Italian study was performed on nQuery (v7.0; Statistical Solutions, Boston, MA). However, for the purposes of this pilot study to assess feasibility of screening for GD in a general adult Haematology outpatient setting, we limited the assessment to achieving half the enrolment in a one year period. Descriptive statistics were used for secondary analyses.

\section{Ethical obligations}

This study was approved by local Ethics Review Board, the Western University Health Science Research Ethics Board, No. 106857. Patients fulfilling the inclusion criteria provided written informed consent. Positive DBS tests were communicated to the MRP and standard of care practices regarding patient management was appropriated.

\section{Results}

In one year of enrolment, from November 2015 to November 2016, 221 patients were screened for GD which surpassed our expectations for enrolling patients. Enrolment occurred weekly in 1 of 3 general non-malignant adult haematology clinics with each clinic being approximately one half-day in duration. The average age of patients was 75.8 years, range (18 to 92). Twenty-one percent had splenomegaly, $96 \%$ had thrombocytopenia, and $20.8 \%$ had both splenomegaly and thrombocytopenia (Table 1). Imaging studies to assess spleen size was performed on $60.1 \%$ of the patients. Of the 210 patients who screened negative for GD, the mean GBS result was $9.19 \mathrm{pmol} / \mathrm{punch} /$ hour. Eleven of 221 patients screened had an initial positive or borderline $(<4.45 \mathrm{pmol} /$ punch/hour $)$ screen on the DBS test. Nearly all ( 9 of 11 ) were Caucasian and $73 \%$ were male. Splenomegaly was not a prominent feature in this group (18\%). None of these patients had a prior splenectomy.

\begin{tabular}{|c|c|c|}
\hline & $\begin{array}{l}\text { Patients with Reduced } \\
\text { DBS Activity }(n=11)\end{array}$ & $\begin{array}{l}\text { Patients with Normal } \\
\text { GBS Activity }(n=210)\end{array}$ \\
\hline \multicolumn{3}{|l|}{ Gender } \\
\hline Male & 8 & 90 \\
\hline Female & 3 & 120 \\
\hline Ethnicity (C/A/O) & $9 / 1 / 1$ & $192 / 2 / 16$ \\
\hline Age, mean [18-92] & 59.4 & 76.7 \\
\hline \multicolumn{3}{|l|}{ Inclusion criteria } \\
\hline Splenomegaly & $2(18 \%)$ & $44(21 \%)$ \\
\hline Thrombocytopenia & $9(81 \%)$ & $204(97 \%)$ \\
\hline Anemia & $2(18 \%)$ & $76(36 \%)$ \\
\hline Bone pain & $2(18 \%)$ & $16(7 \%)$ \\
\hline MGUS & $0(0 \%)$ & $7(3 \%)$ \\
\hline Splenectomy & $0(0 \%)$ & $27(13 \%)$ \\
\hline \multicolumn{3}{|c|}{ Diagnosis of splenomegaly } \\
\hline Abdominal US & $1(9 \%)$ & $84(40 \%)$ \\
\hline Abdominal CT & $1(9 \%)$ & $38(18 \%)$ \\
\hline Pathological specimen & $0(0 \%)$ & $10(5 \%)$ \\
\hline History of fracture & $2(18 \%)$ & $25(12 \%)$ \\
\hline History of hemorrhage & $3(27 \%)$ & $28(13 \%)$ \\
\hline \multicolumn{3}{|c|}{$\begin{array}{l}\text { C: Caucasian, A: Asian, O: Other, MGUS: monoclonal gammopathy of unknown } \\
\text { significance }\end{array}$} \\
\hline
\end{tabular}

Table 1: Patient characteristics and inclusion criteria

The other hallmark clinical manifestations of GD including haemorrhage, bone pain, fractures, and anaemia were not compared to the GBS negative group. Table 2 details the results in the 11 patients 
Page 3 of 4

who tested positive on GBS screening. One patient who had a positive screen was lost to follow-up for confirmatory testing (patient 1 ). Three of the 11 had a repeat positive test (patient $3,5,9$ ) but leukocyte enzyme activity was normal in 2 of these (patient 5,9 ). Two patients were identified as heterozygotes: Patient 3 had a repeat positive test, but did not get a leukocyte enzyme activity tested. This patient was found to have the pathogenic mutation $\mathrm{c} .1504 \mathrm{C}>\mathrm{T}$ missense variant in the GBA gene (also known as the $\mathrm{R} 463 \mathrm{C}$ variant). The other heterozygote patient, patient 11 , had the pathogenic mutation $\mathrm{c}$. [1448T >C; 1483G $>C ; 1497 \mathrm{G}>\mathrm{C}$ ], known as the RecNcil allele.

\section{Discussion}

GD is the most common lysosomal storage disease disorder although it remains relatively rare with an estimated prevalence of 1 in 75,000 [1]. Motta et al. [5] demonstrated a prevalence of 3.6\% when focusing on a selected Italian population with thrombocytopenia and/or splenomegaly. To our knowledge, this is the first time their protocol has been replicated at a North American haematology referral centre. Although we used similar selection criteria and the same DBS assay, we did not identify any patients with GD of the 221 patients screened in this initial feasibility cohort. However, we did find two individuals who were heterozygous carriers of the common GD1 mutation. This may have screening implications for identifying family members who may have GD. Despite the geographic and genetic separation, differences between the Italian and North American study patient populations may explain the observed difference in results. Motta et al. [5] had a large portion of patients with both thrombocytopenia and splenomegaly screened (33.7\%) while only $20.8 \%$ in our study had both those traits. Additionally, we had a far greater proportion of patients with thrombocytopenia alone being screened (97\%) compared to the Italian study (37\%). However, splenomegaly may have been under reported in our study because nearly $40 \%$ of patients did not have imaging studies. Lastly, our results failed to reach statistical power of 336 patients required to achieve $95 \%$ confidence. GD1 commonly presents with hepatosplenomegaly, thrombocytopenia, anaemia, and bone pain [1] placing haematologist directly in line to make the diagnosis. Despite this, a recent survey of 406 haematologists from seven different countries revealed only 1 in 5 physicians considered GD on the differential diagnosis in a patient presenting with all 5 cardinal features: Splenomegaly, thrombocytopenia, anaemia, hepatomegaly, and bone pain [8]. GD1 has traditionally been quoted to have a prevalence of 1 in 75,000 births [1], but this may be an underestimate for the following reasons. First, the diagnosis was difficult to make historically. Up until the 1970s, diagnosis required demonstration of reduced or absent $\beta$-glucosidase activity in cultured blood leukocytes or skin fibroblasts [9]. Since this assay was technically difficult, it was commonly restricted only to tertiary academic hospitals. Furthermore, fragility of blood leukocytes to temperature change and storage during shipping from peripheral centres often made results inaccurate. Second, no effective, approved treatment existed for GD before 1991. This early laboratory difficulty combined with lack of approved therapy probability deterred most physicians from pursuing clinical suspicions of GD in patients. However, both of these hurdles have been overcome. In 1978, the Beutler assay, using an artificial fluorogenic $\beta$-glucosidase substrate on a fixed matrix, did away with leukocyte culturing and replaced it with a drop of blood on a piece of filter paper, making GD testing widely available $[1,7,10,11]$. Then, in 1991 the first glucocerebrosidase enzyme replacement, Alglucerase, was approved in the United States for treatment of GD1 [1]. With faster and easier bedside tests available, clinicians' awareness for GD is rising. This is reflected in the International Collaborative Gaucher Group (ICGG) Gaucher Registry markedly increasing worldwide enrolment over the past 10 years [12].

\begin{tabular}{|c|c|c|c|c|}
\hline Patient No. & $\begin{array}{l}\text { Initial GBS value(pmol/ } \\
\text { punch-1/h-1) }\end{array}$ & $\begin{array}{l}\text { Repeated GBS value(pmol/punch-1/ } \\
\text { h-1) }\end{array}$ & $\begin{array}{l}\text { Leukocytes } \quad \text { beta-Glucocerebrosidase }{ }^{*} \\
(\mathrm{nmol} / \mathrm{mg} / \mathrm{h})\end{array}$ & Genotype1 \\
\hline 1 & 4.36 & NA & NA & NA \\
\hline 2 & 3.14 & 5.27 & 6.8 & \\
\hline 3 & 2.97 & 3.62 & NA & Heterozygote \\
\hline 4 & 3.67 & NA & 6.8 & \\
\hline 5 & 3.7 & 3.6 & 11.4 & \\
\hline 6 & 2.87 & 5.46 & 6.3 & \\
\hline 7 & 4.27 & 8.65 & 8 & \\
\hline 8 & 3.58 & 5.65 & 8.4 & \\
\hline 9 & 4.41 & 4.2 & 8.4 & \\
\hline 10 & 4 & 6.59 & 9.9 & \\
\hline 11 & 3.96 & 4.91 & 5.7 & Heterozygote \\
\hline \multicolumn{5}{|c|}{$\begin{array}{l}\text { * Leukocyte beta-Glucocerebrosidase normal range } 5.0 \text { to } 11.3 \mathrm{nmol} / \mathrm{mg} / \mathrm{h} \\
\text { 1The mutations are described according the traditional amino acid residue numbering. } \\
\text { Patient } 1 \text { was lost to follow-up after initial screening. } \\
\text { Patient } 3 \text { is heterozygote for c. } 1504 \mathrm{C}>\mathrm{T} \text {. } \\
\text { Patient } 5 \text { died of a myocardial infarction and Klebsiella bacteremia and his sample was }\end{array}$} \\
\hline
\end{tabular}


Citation: $\quad$ Russell SA, Sholzberg M, Mangel J, Keeney M, Hedley B, et al. (2018) Gaucher Disease Screening at a General Adult Haematology

Patient 11 is heterozygote for c.[1448T>C; $1483 \mathrm{G}>\mathrm{C} ; 1497 \mathrm{G}>\mathrm{C}]$.

Table 2: GBS positive screened patient profiles.

In conclusion, what this study has demonstrated is the feasibility of screening high risk patients for GD. Drawing from only 3 benign hematology clinics (approximately 3 half-days per week), over 200 patients were able to be screened within one year [13-16]. As we plan to expand our enrolment to other North American centres, this number should increase substantially which will facilitate exploration of numerous hypothesis and will definitively assess the clinical relevance of this diagnostic entity in North American patients with thrombocytopenia.

\section{Acknowledgements}

Contributors: S.A.R. and C.C.H. designed and implemented the study. S.A.R. completed the data collection, data analysis and initial manuscript write-up. C.C.H. revised the manuscript.

Funders: Genzyme Corporation

Prior presentations: Preliminary data presented as an abstract at the Department of Medicine, Internal Medicine Resident Research Day 2017, Western University, London Ontario.

\section{Conflict of Interest}

C.C.H. has received speaker's honorarium from Genzyme.

M.S. has received unrestricted research support from Genzyme.

\section{References}

1. Rosenbloom BE, Weinreb NJ (2013) Gaucher disease: a comprehensive review. Crit Rev Oncog 18: 163-175.

2. Beulter E (2001) Gaucher's Disease. The Metabolic and molecular basis of inherited disease. NewYork: McGraw-Hill. 3635-3668.

3. Horowitz M (1989) The human glucocerebrosidase gene and pseudogene: structure and evolution. Genomics 4: 87-96.
4. Weinreb NJ, Kaplan P (2015) The history and accomplishments of the ICGG Gaucher registry. Am J Hematol 90: S2-5.

5. Motta I (2015) A multicentre observational study for early diagnosis of Gaucher disease in patients with Splenomegaly and/or Thrombocytopenia. Eur J Haematol 96: 352-359.

6. Olivova P (2008) An improved high-throughput dried blood spot screening method for Gaucher disease. Clin Chim Acta 398: 163-164.

7. Stroppiano M (2014) Validity of $\beta$-D-glucosidase activity measured in dried blood samples for detection of potential Gaucher disease patients. Clin Biochem 47: 1293-1296.

8. Mistry PK (2007) Consequences of diagnostic delays in type 1 Gaucher disease: the need for greater awareness among hematologists-oncologists and an opportunity for early diagnosis and intervention. Am J Hematol 82: 697-701.

9. Beutler E, Kuhl W (1970) The diagnosis of the adult type of Gaucher's disease and its carrier state by demonstration of deficiency of betaglucosidase activity in peripheral blood leukocytes. J Lab Clin Med 76: 747-755.

10. Chamoles NA (2002) Gaucher and Niemann-Pick diseases--enzymatic diagnosis in dried blood spots on filter paper: retrospective diagnoses in newborn-screening cards. Clin Chim Acta 317: 191-197.

11. Wenger DA (1978) Synthetic substrate beta-glucosidase activity in leukocytes: a reproducible method for the identification of patients and carriers of Gaucher's disease. Clin Genet 13: 145-153.

12. Weinreb NJ (2008) Life expectancy in Gaucher disease type 1. Am J Hematol 83: 896-900.

13. Grabowski GA (2005) Recent clinical progress in Gaucher disease. Curr Opin Pediatr 17: 519-524.

14. Harris CM, Taylor DS, Vellodi A (1999) Ocular motor abnormalities in Gaucher disease. Neuropediatrics 30: 289-293.

15. Thomas AS, Mehta AB, Hughes DA (2013) Diagnosing Gaucher disease: an on-going need for increased awareness amongst haematologists. Blood Cells Mol Dis 50: 212-127.

16. Mistry PK (2011) A reappraisal of Gaucher disease-diagnosis and disease management algorithms. Am J Hematol 86: 110-115. 